\title{
TaAMT2;3a, a wheat AMT2-type ammonium transporter, facilitates the infection of stripe rust fungus on wheat
}

\author{
Junpeng Jiang ${ }^{1 \dagger}$, Jing Zhao ${ }^{2 \dagger}$, Wanlu Duan², Song Tian², Xiaodong Wang ${ }^{2}$, Hua Zhuang ${ }^{2}$, Jing Fu ${ }^{1 *}$ (D) and
} Zhensheng Kang ${ }^{2^{*}}$

\begin{abstract}
Background: Ammonium transporters (AMTs), a family of proteins transporting ammonium salt and its analogues, have been studied in many aspects. Although numerous studies have found that ammonium affects the interaction between plants and pathogens, the role of AMTs remains largely unknown, especially that of the AMT2-type AMTs.

Results: In the present study, we found that the concentration of ammonium in wheat leaves decreased after infection with Puccinia striiformis f. sp. tritici (Pst), the causal agent of stripe rust. Then, an AMT2-type ammonium transporter gene induced by Pst was identified and designated as TaAMT2;3a. Transient expression assays indicated that TaAMT2;3a was located to the cell and nuclear membranes. TaAMT2;3a successfully complemented the function of a yeast mutant defective in $\mathrm{NH}_{4}{ }^{+}$transport, indicating its ammonium transport capacity. Function of TaAMT2;3a in wheat-Pst interaction was further analyzed by barley stripe mosaic virus (BSMV)-induced gene silencing. Pst growth was significantly retarded in TaAMT2;3a-knockdown plants, in which ammonium in leaves were shown to be induced at the early stage of infection. Histological observation showed that the hyphal length, the number of hyphal branches and haustorial mother cells decreased in the TaAMT2;3a knockdown plants, leading to the impeded growth of rust pathogens.
\end{abstract}

Conclusions: The results clearly indicate that the induction of AMT2-type ammonium transporter gene TaAMT2;3a may facilitates the nitrogen uptake from wheat leaves by Pst, thereby contribute to the infection of rust fungi.

Keywords: Puccinia striiformis f.sp. tritici, Ammonium transporter, TaAMT2;3a, Stripe rust

\section{Background}

Nitrogen is an indispensable element for all living organisms, and its uptake and utilization are the major limiting factors for plant growth and crop yield [1, 2]. As an essential nutrient, nitrogen is the key component of biological macromolecules such as proteins, nucleic acids, vitamins and hormones. Thus, uptake and utilization of nitrogen affect nearly all biological processes and are tightly regulated $[3,4]$.

\footnotetext{
* Correspondence: jingfu@nwsuaf.edu.cn; kangzs@nwsuaf.edu.cn

†Junpeng Jiang and Jing Zhao contributed equally to this work.

'State Key Laboratory of Crop Stress Biology for Arid Areas and College of Life Sciences, Northwest A\&F University, Yangling, Shaanxi, People's Republic of China

${ }^{2}$ State Key Laboratory of Crop Stress Biology for Arid Areas and College of Plant Protection, Northwest A\&F University, Yangling, Shaanxi, People's Republic of China
}

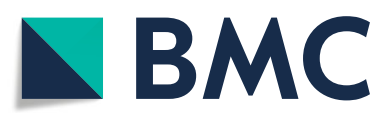

(c) The Author(s). 2019 Open Access This article is distributed under the terms of the Creative Commons Attribution 4.0 International License (http://creativecommons.org/licenses/by/4.0/), which permits unrestricted use, distribution, and reproduction in any medium, provided you give appropriate credit to the original author(s) and the source, provide a link to the Creative Commons license, and indicate if changes were made. The Creative Commons Public Domain Dedication waiver (http://creativecommons.org/publicdomain/zero/1.0/) applies to the data made available in this article, unless otherwise stated. transported into plant root cells by AMTs and NRTs (nitrate transporters) respectively, are the main plant available inorganic forms of nitrogen in soils. Because $\mathrm{NO}_{3}{ }^{-}$ needs to be reduced to $\mathrm{NH}_{4}{ }^{+}$before assimilation in plant cells, $\mathrm{NH}_{4}{ }^{+}$is usually more preferentially absorbed than nitrate $[1,5]$. After transport into root cells, $\mathrm{NH}_{4}{ }^{+}$is assimilated into glutamate via the glutamine synthase/glutamate synthase (GS/GOGAT) cycle for the further synthesis of organic macromolecules.

Plant ammonium transporters belong to multigene families. In Arabidopsis and rice, there are 6 and 10 AMTs, respectively, whereas at least 54 AMT homologues are present in the wheat genome [6,7]. According to their sequence and phylogeny, these AMTs are categorized into two subfamilies, AMT1 and AMT2. 
AMT1-type AMTs are mainly expressed in roots and play a critical role in ammonium uptake from the soil [8]. Out of the six AMTs in Arabidopsis, four AMT1-type transporters contribute to ammonium uptake in root cells. An AMT1;1 AMT1;2 AMT1;3 AMT2;1 quadruple deletion line $(q k o)$ showed significantly impaired ammonium uptake ability and severe growth depression under ammonium supply [9]. AMT2-type AMTs are usually expressed in various plant tissues, including roots, shoots and leaves at relative low levels. Compared with the well-studied AMT1-type transporters, AMT2-type AMTs are not able to transport ${ }^{14} \mathrm{C}$-Methylamine (an analog of ammonia), which make it difficult to characterize their transport kinetics and physiological roles [10].

It has long been known that the nitrogen status and availability in plants affect the disease process [5]. A considerable number of studies have shown that a high nitrogen level enhances plant susceptibility to biotrophic and hemibiotrophic pathogens, known as nitrogen-induced susceptibility (NIS) [11]. It has been suggested that a high concentration of nitrogen in the host is favourable for nutrient acquisition by pathogens. On the other hand, biotrophic and hemibiotrophic pathogens often attempt to manipulate plant metabolism to their advantage. The induction of host genes related to nitrogen uptake and transport during infection is one of the most common way employed by pathogens. Three wheat ammonium transporter genes, TaAMT1;1a, TaAMT1;1b and TaAMT1; $3 a$, are induced only in compatible interactions between wheat and stem rust fungus [12]. Similarly, the expression of the nitrate transporter gene NRT2.6 is induced after the inoculation of Arabidopsis thaliana by the phytopathogenic bacterium Erwinia amylovora [13].

Stripe rust is one of the most serious diseases threatening the growth and production of wheat worldwide. The causal agent, Puccinia striiformis f. sp. tritici (Pst), is a typical obligate biotrophic fungus. The nutrients required for the growth and reproduction of Pst come entirely from wheat via haustoria, the special fungal structures formed in the host mesophyll cells [14]. Thus, for rust fungi, uptake of sufficient nutrients is the determinant for successful colonization. Several genes related to sucrose transport and metabolism have been identified and are well documented [15-17]. It has also been demonstrated that leaf nitrogen content is important for sustaining the stripe rust epidemics during winter [18]. Moreover, nitrogen availability in the host leaves significantly affected spore production for wheat leaf rust fungus [19], indicating a direct role of host nitrogen in rust disease. However, it is not well understood how rust fungi manipulate host nitrogen metabolism and acquire nitrogen from the host.

In this study, we analysed the changes in ammonium content in different tissues of wheat infected by stripe rust and identified an AMT2-type wheat gene, TaAMT2; $3 a$, induced by Pst infection. Then, the subcellular localization of TaAMT2;3a was examined by transient expression of GFP fusion protein, and its ammonium transport activity was validated by yeast mutant complementary assay. In addition, the function of the TaAMT2; $3 a$ in the wheat-Pst interaction was analysed using a genetic silencing technique through barley stripe mosaic virus.

\section{Results}

Determination of ammonium contents in specific tissues of wheat infected by Pst

During the interaction between Pst and wheat, the pathogen need to uptake nitrogen from the host to support its growth and multiplication. To access the change in ammonium contents in wheat infected by Pst, we measured the ammonium concentrations in roots, stems and leaves at $0,12,24,36,48,72$ and $120 \mathrm{~h}$ post inoculation (hpi). The ammonium contents between inoculated wheat and non-inoculated control were compared. The results demonstrated that the relative ammonium contents in the roots increased as early as $12 \mathrm{hpi}$ and were maintained at a high level throughout the infection. In contrast, the relative ammonium contents in the leaves and stems significantly decreased, especially in the leaves, and reached the lowest point at $12 \mathrm{hpi}$ and 36 hpi, respectively (Fig. 1a). These results revealed that the infection by stripe rust fungus greatly affected the transport and metabolism of ammonium in wheat, which led to a decline in ammonium levels in the leaves and stems and an increase in the roots.

\section{Identification of the wheat AMT2-type AMT gene TaAMT2;3a induced by Pst infection}

The fluctuation of ammonium contents during Pst infection might be related to the ammonium transportation. To identify wheat genes involved in ammonium metabolism and transport during Pst infection, we screened AMT genes that were differentially expressed during wheat-Pst interaction using the transcriptome database [20]. A wheat gene encoding an AMT2-type ammonium transporter was found to be upregulated upon Pst infection. This gene was designated as TaAMT2;3a according to its homologous gene in Arabidopsis and a published reference [12].

The induction of TaAMT2;3a by Pst infection was further confirmed by quantitative RT-PCR. Analysis of TaAMT2;3a expression revealed that during a compatible interaction, the transcription of TaAMT2;3a was induced as early as $6 \mathrm{hpi}$ and reached the highest level at $18 \mathrm{hpi}$, which was 7.3-fold higher than that in the control plants (Fig. 1b). The mRNA levels of TaAMT2;3a in different wheat tissues (roots, stems, leaves) were 

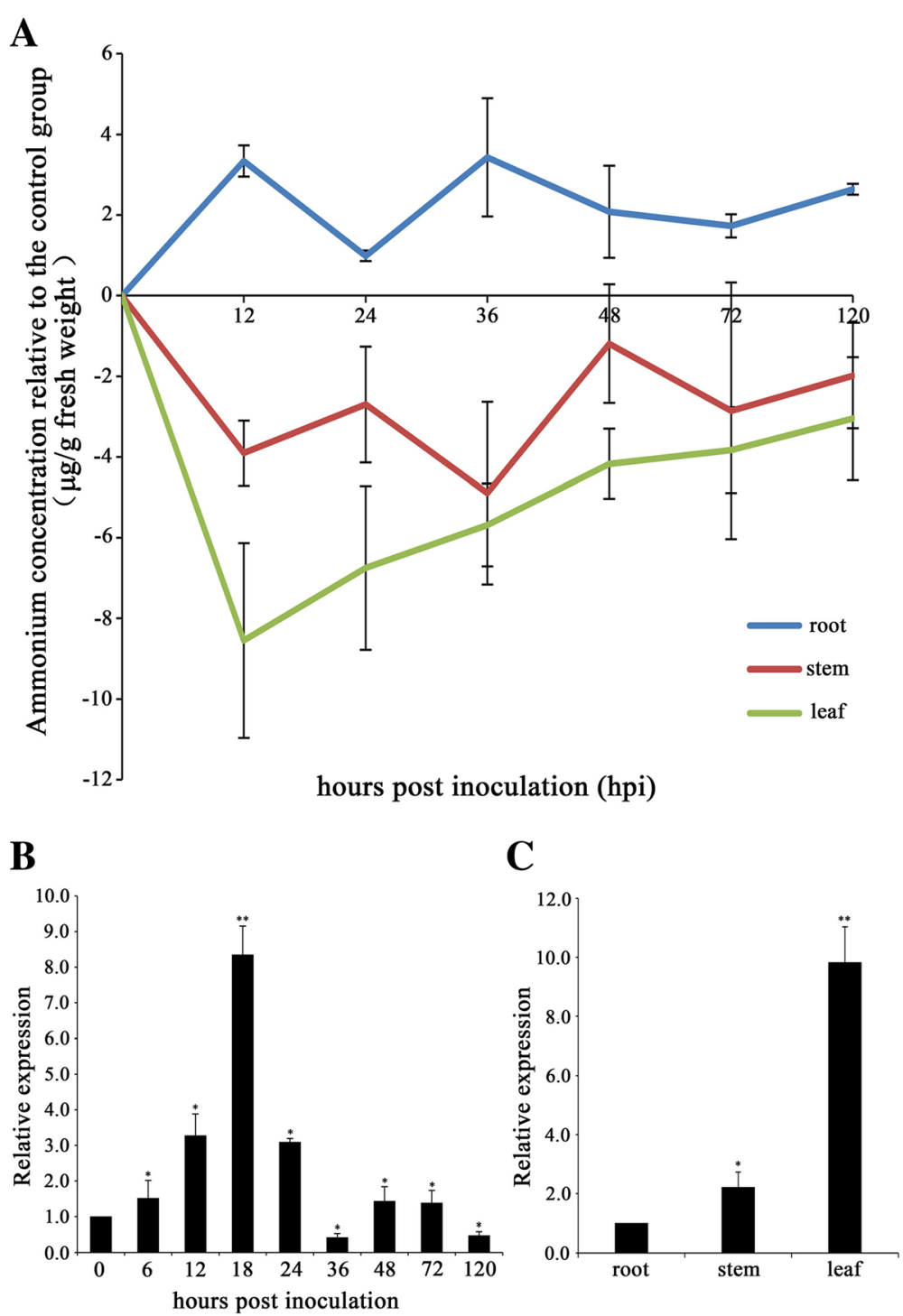

Fig. 1 Relative ammonium contents in different wheat tissues after inoculation with Puccinia striiformis f.sp. tritici (Pst) and the expression pattern of TaAMT2:3a. a Relative ammonium concentrations in leaves, stems, and roots from wheats inoculated with Pst at different time points. The relative ammonium concentration was calculated by subtracting the ammonium concentration of the non-inoculated control plants from that of the inoculated plants. b Relative transcription levels of the TaAMT2;3a gene in wheat leaves inoculated with Pst compared with that in the control plants. c The expression pattern of the TaAMT2;3a gene in different tissues. The data were normalized to the wheat TaEF-1a gene. All the results were obtained from three independent replicates. Asterisks indicate significant differences from 0 hpi using Student's t-test. $(P<0.05)$

examined. The results indicated that TaAMT2;3a was detectable in all tested wheat tissues, and the transcription levels of TaAMT2;3a in leaves were significantly higher than those in roots and stems (Fig. 1c). These results suggested that TaAMT2;3a may be involved in transporting ammonium in the leaves and play an important role in the wheat-Pst interaction.

The full-length CDS (1449 bp) of the TaAMT2;3a gene was isolated from wheat variety Suwon11. Sequence alignment with orthologues in the wheat variety Chinese Spring genome from the IWGSC (International Wheat Genome Sequencing Consortium) database showed that there are three homoeologous genes located on chromosomes $1 \mathrm{~A}, 1 \mathrm{~B}$ and $1 \mathrm{D}$. The TaAMT2;3a sequence was identical to the sequence on the long arm of chromosome 1D (Additional file 1: Figure S1).

TaAMT2;3a is a typical ammonium transporter containing a signal peptide, an ammonium transporter region (from 21 to $437 \mathrm{aa}$ ) and a C-terminal region (CTR) (Fig. 2a, Additional file 2: Figure S2). Multiple sequence alignment showed that TaAMT2;3a shares the highest identity (83\%) with OsAMT2;1 from rice, followed by AtAMT2;1 from Arabidopsis, with 71\% identity. To better understand the evolutionary relationship between 


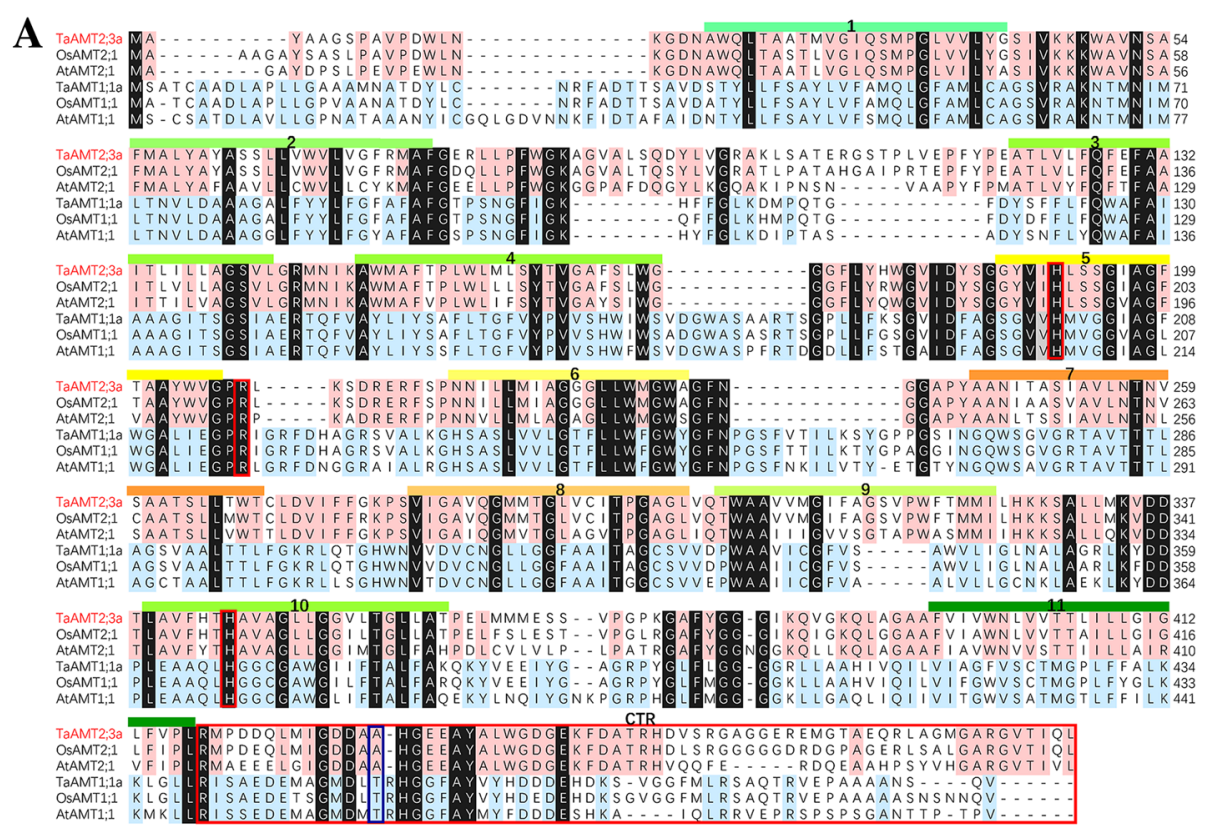

B

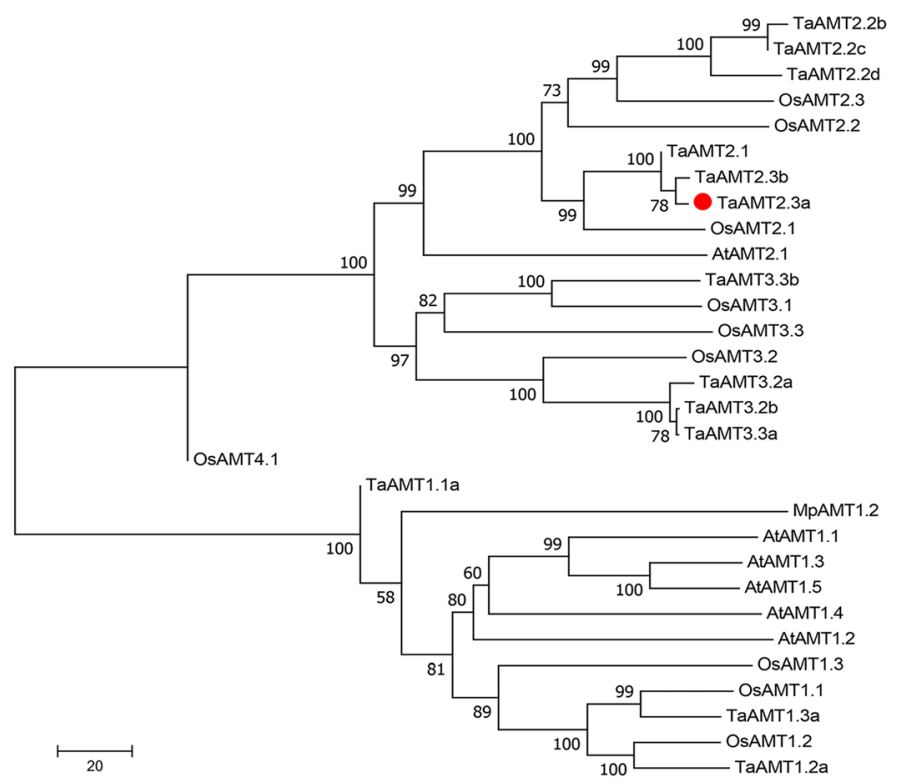

Fig. 2 Multiple sequence alignment and phylogenetic analysis of TаAMT2;3a. a Multiple sequence alignment of amino acid sequences from Arabidopsis thaliana (AtAMT1;1, AtAMT2;1), Oryza sativa (OsAMT1;1, OsAMT2;1) and Triticum aestivum (TaAMT2;3a). Ammonium transporter domains: red line. CTR: black line. Highly conserved domain: blue linear box. b Phylogenetic analysis of TaAMT2;3a and homologues in wheat and other plant species. At: Arabidopsis thaliana; Ta: Triticum aestivum; Mp: Marchantia polymorpha; and Os: Oryza sativa. Multiple sequence alignments and the neighbour joining tree were created using the ClustalW algorithm by MEGA (version 7.0)

TaAMT2;3a and its homologues from other plant species, a phylogenetic tree was constructed based on the full-length amino acid sequences from wheat, rice, Arabidopsis and liverwort (Fig. 2b and Additional file 3: Table S1). Phylogenetic analysis demonstrated that except for OsAMT4;1, other AMTs were unambiguously categorized into two subfamilies, AMT1-type and AMT2-type AMTs. TaAMT2;3a was clustered into the AMT2 group and had the closest evolutionary relationship with OsAMT2.

TaAMT2;3a is localized to the cytosolic and nuclear membranes in wheat cells and complement yeast ammonium transporter mutant

Previous studies of AMTs in other organisms have reported that AMTs are mainly located on cell membranes 
$[12,21]$. In the current study, transient expression of the TaAMT2;3a-GFP fusion protein was used to determine the subcellular localization of TaAMT2;3a in wheat protoplasts. Compared with the ubiquitous distribution of GFP in the control group, the fluorescence of the TaAMT2;3a-GFP fusion protein was mainly distributed on the cell and nuclear membranes of wheat cells (Fig. 3a). Thus, the results confirmed that TaAMT2;3a was localized to the cell and nuclear membranes in wheat cells.

To further characterize the biochemical function of TaAMT2;3a, we tested the ability of TaAMT2;3a to transport $\mathrm{NH}_{4}{ }^{+}$. The TaAMT2;3a protein was expressed in the yeast triple $M e p$ deletion strain 31019b (mep1, mep2, mep3, ura3), which is entirely defective in $\mathrm{NH}_{4}{ }^{+}$transport and unable to grow on medium containing $<5 \mathrm{mM} \mathrm{NH}_{4}{ }^{+}$as the sole nitrogen source [21]. The results showed that the yeast mutant transformed with TaAMT2;3a grew on medium containing 1 or $5 \mathrm{mM} \mathrm{NH}_{4} \mathrm{Cl}$ as the sole nitrogen source (Fig. 3b). This finding indicates that TaAMT2;3a complements the $\mathrm{NH}_{4}{ }^{+}$uptake defect of $31019 \mathrm{~b}$ and is likely responsible for high-affinity ammonium transport in plants.

\section{Silencing of TaAMT2;3a impairs fungus pathogenicity} during wheat-Pst interaction

To reveal the function of the TaAMT2;3a gene in the wheat-Pst interaction, barley stripe mosaic virus (BSMV)-induced gene silencing (VIGS) was applied. Due to the high similarity among the three TaAMT2;3a homoeologs in the wheat $\mathrm{A}, \mathrm{B}$, and $\mathrm{D}$ genomes, two fragments were designed to silence the three homoeologs together (Additional file 1: Figure S1). The silencing of the wheat phytoene desaturase gene (PDS) was used as the positive control for the gene silencing system to confirm whether the VIGS system functions correctly. Mild chlorotic mosaic symptoms appeared on the fourth leaves in all plants at 10 days after inoculation with BSMV. The BSMV::TaPDS-inoculated plants exhibited strong photobleaching symptoms, suggesting that the VIGS system worked well (Fig. 4a). After inoculation with Pst, both TaAMT2;3a-knockdown wheat plants (BSMV::TaAMT2;3a -1as and BSMV:: TaAMT2;3a -2as) exhibited impaired fungal development compared to the controls (Fig. 4b). Quantitative RT-PCR analyses confirmed the suppression of TaAMT2;3a transcription in wheat plants preinoculated with virus (Fig. 4c).

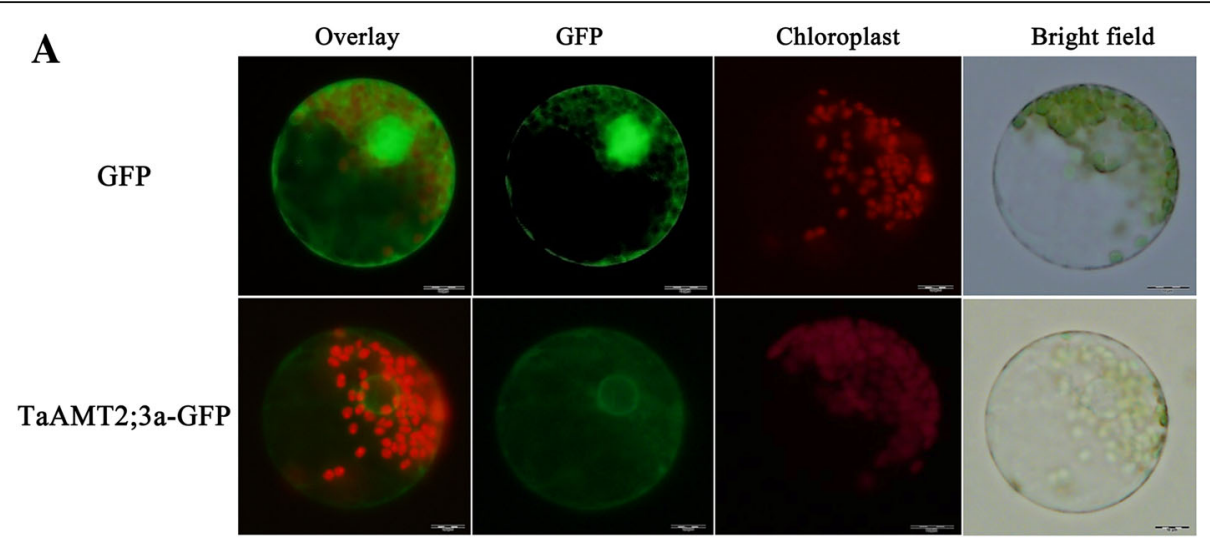

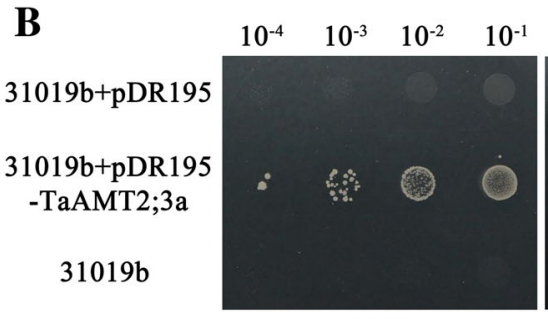

$1 \mathrm{mM} \mathrm{NH}_{4} \mathrm{Cl}$

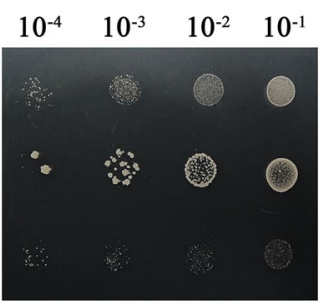

$5 \mathrm{mM} \mathrm{NH}_{4} \mathrm{Cl}$

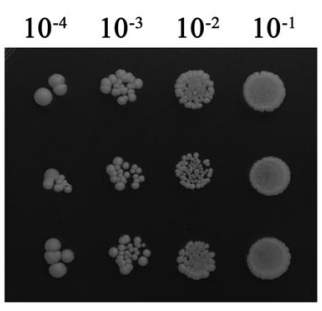

$1 \mathrm{mM}$ Arginine

Fig. 3 Subcellular localization and functional complementation analysis of TaAMT2;3a. a Subcellular localization of TaAMT2;3a in wheat protoplasts. Wheat protoplasts were transformed with a plasmid carrying a eGFP-TaAMT2;3a fusion protein. The picture was obtained using a fluorescence microscope. The top four images indicate GFP (control), and the bottom four images indicate eGFP-TaAMT2;3a. Bar: 10 $\mu$ m. b Functional complementation analysis of TaAMT2;3a in yeast ammonium transporter mutant. Yeast mutant strain 31019b (mep1, mep2, mep3, ura3) cells were transformed with a pDR195 vector expressing TaAMT2;3a and tested for growth on media supplemented with 1 mM NH $\mathrm{Cl}_{4} 5 \mathrm{mM}$ $\mathrm{NH}_{4} \mathrm{Cl}$ and $1 \mathrm{mM}$ arginine (Arg). The empty vector (pDR195) was used as the control 
A

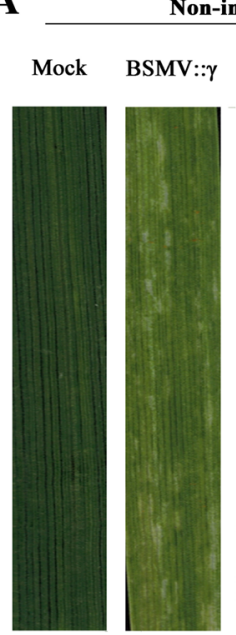

Non-inoculated with $P$ st BSMV:: BSMV
TaAMT2; PDS -1 as
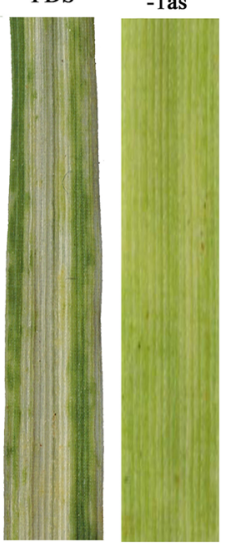

C

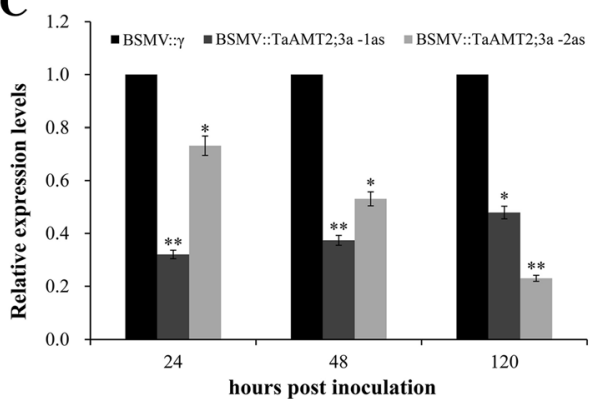

B

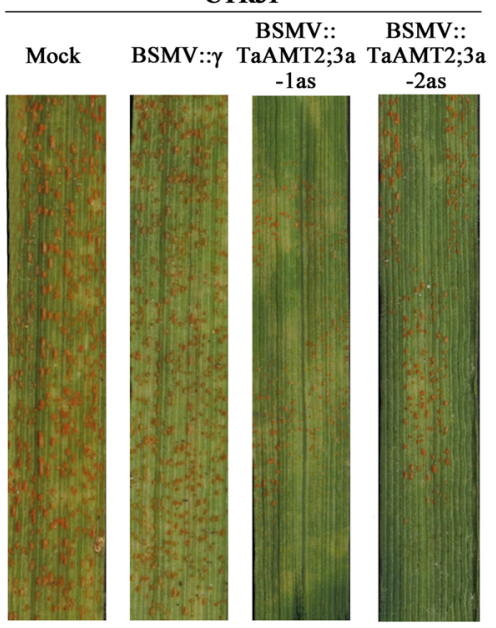

D

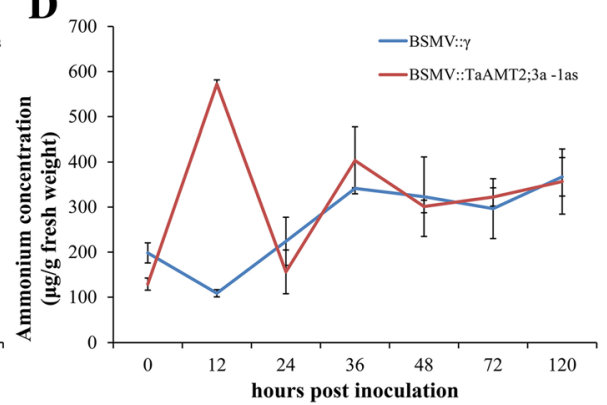

Fig. 4 Functional analysis of TaAMT2;3a in wheat-Pst interaction using a virus-induced gene silencing (VIGS) system. a Symptoms of wheat leaves treated by recombinant virus BSMV::Y, BSMV::PDS, BSMV::TaAMT2;3a-1as and BSMV::TaAMT2;3a -2as at 10 dpi. Mock leaves were treated only with FES buffer. $\mathbf{b}$ The phenotype of the fourth leaves inoculated with Pst virulent strain CYR31 at 14 dpi. c Relative transcription levels of TaAMT2;3asilencing plants after inoculated with Pst. The data were normalized to the TaEF-1a expression level. The transcription levels of TaAMT2;3a in wheat leaves treated by BSMV::y at each time point were set to one. (D) Ammonium concentrations in leaves from TaAMT2;3a-knockdown and control wheat plants inoculated with Pst. Error bars represent variations among three independent replicates. Asterisks $\left(^{*}\right.$ and $\left.{ }^{* *}\right)$ indicate a significant differences from 0 hpi using Student's t-test $(P<0.05$ and 0.01 , respectively)

The ammonium concentrations of leaves from TaAMT2;3a-knockdown plants as well as those from the control plants were measured. The results showed that the ammonium concentrations in leaves from the TaAMT2;3a-knockdown plants is far more than that from the control plants at $12 \mathrm{hpi}$, while no apparent difference were observed after 24 hpi (Fig. 4d). These results suggested that suppression of TaAMT2;3a gene greatly affected the ammonium transportation at the early stage of Pst infection, and retarded the growth of the fungi.

\section{Histological observation of Pst growth in TaAMT2;3a- knockdown plants}

Leaf samples from TaAMT2;3a-knockdown plants were examined by fluorescence microscopy to illustrate fungal development (Fig. 5a). As shown in the figure, the number of hyphal branches in wheat leaves from TaAMT2; $3 a$-knockdown plants were obviously less than those from control plants at 48 hpi (Fig. 5b). Additionally, the number of haustorial mother cells and the hyphal length at 24 hpi in leaves from TaAMT2;3a-knockdown plants were much lower than those from control plants (Fig. $5 \mathrm{c}$ and d). For the infection site area, no significant change was observed until 120 hpi (Fig. 5e). Altogether, the overall histological results indicate that suppression of TaAMT2;3a expression restricts fungal growth and ultimately affects sporulation and the pathogenicity phenotype.

\section{Discussion}

As a main nitrogen source, ammonium plays an important role in plant growth and development. Knowledge about ammonium uptake and translocation has been obtained by extensive investigations on ammonium transporters in Arabidopsis and rice. However, the physiological roles of AMTs in the interaction between plants and environmental microbes remain largely unknown. 

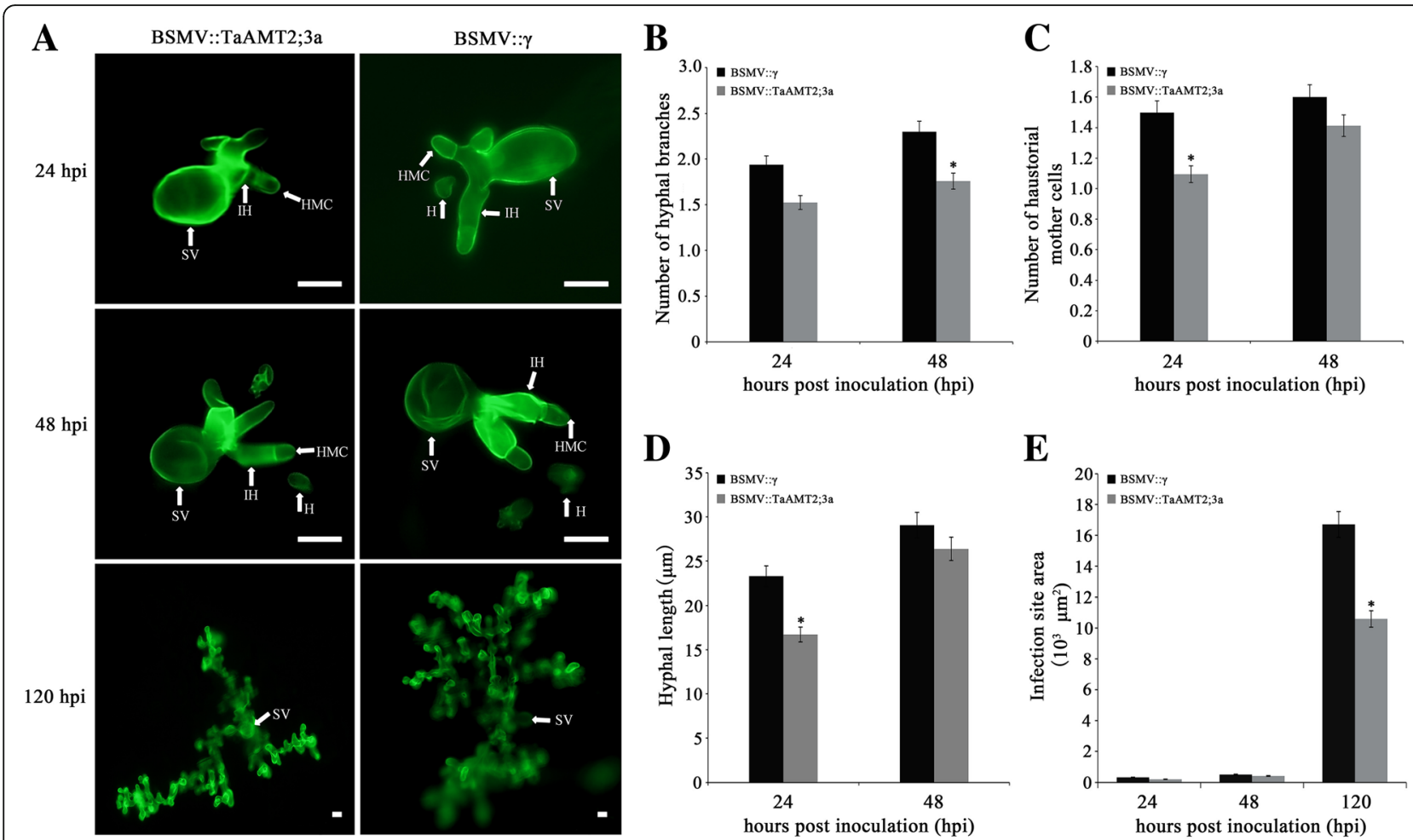

Fig. 5 Histological observation of fungi growth in TaAMT2; 3a-knockdown plants challenged by Pst. a Leaves inoculated with Pst were observed at 24, 48 and 120 hpi. The infected sites were observed by fluorescence microscopy. H: haustorium; HMC: haustorial mother cell; SV: substomatal vesicle; IH: infection hyphae. Bar, 50 mm; The number of hyphal branches (b), the number of haustorial mother cells (c), the hyphal length (d) and the infection site areas (e) in TaAMT2; 3a-knockdown plants were compared with that in control plants. The fungal structures were stained with wheat germ agglutinin (WGA) and each result was considered from 30 infection sites. Asterisks indicate significant difference $(P<0.05)$ from BSMV::y-inoculated plants using Student's t-test

Although two types of AMTs share common secondary protein structures and biochemical activities, AMT2type AMTs showed apparent difference in amino acid sequences, especially in those regions related to transport activity regulation. For example, the T460 residue in Arabidopsis AtAMT1 is conserved in all plant AMT1 proteins, and controls ammonium transport activity through phosphorylation and inactivation by the kinase CIPK23 upon extracellular ammonium exposure [2224]. A phosphomimetic mutation of MpAMT1;2 (T475D) from Marchantia polymorpha also exhibited complete loss of ammonium transport activity [25]. With regards to AMT2-type ammonium transporters, an alanine (A) is substituted for the threonine (T) at the corresponding T460 site of AtAMT1;1. Therefore, it seems that AMT2-type AMTs may play a unique role other than ammonium uptake. Recent studies revealed that an AMT2-type ammonium transporter, AtAMT2.1, not only participates in ammonium uptake, but also plays an important role in the translocation of ammonium from the root to the shoot through xylem loading [26]. In the present study, sequence alignment and phylogenetic analysis indicated that TaAMT2;3a is a typical AMT2-type ammonium transporter. In addition, the high expression levels of TaAMT2;3a in leaves imply that it is likely involved in ammonium translocation in leaves.

As one of the most important executors of nutrient transport, plant AMTs also play key roles in the interactions between plants and microbes, especially obligate biotrophic fungi. An increasing number of studies have demonstrated that plant AMTs are involved in the mutualistic symbiosis of Arbuscular mycorrhizae (AM) fungi. Most of these AMTs belong to the AMT2 subfamily and are highly or exclusively expressed in AM-colonized roots [27]. For example, two sorghum AMT2 genes, SbAMT3;1 and $S b A M T 4$, were induced to express in roots colonized by arbuscular fungi [28]. SbAMT3;1 knockdown plants showed greatly reduced uptake of ammonium from the AM network and less growth stimulation by AM fungal colonization [29]. Except for ammonium transport, some AMT members serve as transceptors that sense as well as transport their substrates. In Medicago colonized by Arbuscular mycorrhizae, AMT2;3 is located in the periarbuscular membrane and is required for the suppression of premature arbuscule degeneration in pad4 mutants under low-nitrogen conditions [30]. 
To date, there are only a few reports related to AMT function in plant-pathogen interactions. The Arabidopsis AMT1.1 mutant displays enhanced resistance against Plectosphaerella cucumerina and reduced susceptibility to Pseudomonas syringae [31]. Another study demonstrated that three AMT1-type members, $T a A M T 1 ; 1 a$, TaAMT1;1b, and TaAMT1;3a, were specifically induced in the compatible interaction between wheat and stem rust fungus (Puccinia graminis f. sp. tritici, Pgt) but not in the incompatible interaction. Additionally, wheat plants grown with $\mathrm{NH}_{4}^{+}$ were more vulnerable to Pgt than plants under $\mathrm{N}$-free conditions, indicating that ammonium transport in root mediated by AMT1-type transporters may facilitate the infection of wheat stem rust [12]. While, the role of AMT2-type transporters in wheat-rust interaction is not clear. In the present study, the increased expression of TaAMT2;3a during Pst infection was expected to accelerate the ammonium translocation from intercellular space to mesophyll cells. However, the result showed that ammonium concentrations in the aerial part of wheat after Pst infection declined, which may be attributed to immediate assimilation of excess ammonium into amino acids [26]. Transcriptional analysis revealed that the single gene encoding AMT in Pst was downregulated during wheat infection, while the genes encoding amino acid transporters were upregulated, suggesting that the main form of nitrogen uptake by Pst from the host is amino acids rather than ammonium [32]. Thus, TaAMT2;3a may be exploited by the rust fungi to meet their demand for nitrogen. This hypothesis was further supported by the retarded fugal growth and increased level of ammonium contents in leaves at the early stage of Pst infection in TaAMT2;3a-knockdown plants.

This is the first report on the function analysis of AMT2-type AMTs during interactions between plants and pathogens. Our results, together with the previous studies [12], suggest that the coordination between ammonium uptake and translocation mediated by AMT1 and AMT2-type AMTs helps rust pathogens uptake and assimilate nitrogen nutrients from the host. However, how and through what mechanism host ammonium or nitrogen is translocated to the fungus is still unclear. Analysis of other ammonium transporters and a more detailed determination of ammonium dynamics in hosts and pathogens are necessary for a full understanding of the biological roles of plant AMTs in plant-pathogen interactions.

\section{Conclusions}

The results obtained by this study indicate that during the wheat-Pst interaction, the wheat AMT2-type gene
TaAMT2;3a was induced by rust infection and may facilitate the nitrogen uptake by rust fungus. The present study not only provides new knowledge on the function of AMT2-type AMTs in plant-pathogen interaction, but also lays a foundation for revealing the mechanism of nitrogen acquisition from host for rust fungi.

\section{Methods \\ Plant and fungal materials, growth conditions and inoculation}

Wheat (Triticum aestivum) variety Suwon11 and a Chinese Pst race CYR31 (virulent to Suwon11) were obtained from the Prof. Zhensheng Kang's Lab (Northwest A\&F University, China) and were used to study the wheat-Pst interaction. Wheat seedlings cultivation and Pst inoculation procedures and conditions were followed as described previously [33]. The fresh urediospores of CYR31 were inoculated on the wheat leaves at the first leaf stage. Parallel mock control plants were inoculated with sterile water. After inoculation, the plants were kept in a dark room at $100 \%$ humidity for $24 \mathrm{~h}$, then transferred to a growth chamber at $14^{\circ} \mathrm{C}$ under a long photoperiod ( $16 \mathrm{~h}$ light $/ 8 \mathrm{~h}$ dark). Wheat leaves were sampled at $0,6,12,18,24,36,48,72$ and 120 h post-inoculation (hpi). Meanwhile, samples from leaf, stem and root were collected for gene expression analysis. The time points were selected based on the microscopic study of the wheat-Pst interaction [34].

\section{Cloning of TaAMT2;3a and sequence analyses}

To clone the TaAMT2;3a gene, primers (Additional file 4: Table S2) was designed based on the sequence from wheat genome sequence using Primer 6.0 software. The amplified fragment of TaAMT2;3a CDS from Suwon11 was cloned into the pMD18-T simple vector and sequenced. This cloned sequence was then aligned with the wheat cv. Chinese Spring genome, based on the data of International Wheat Genome Sequencing Consortium (https://urgi.versailles.inra.fr/blast/). The chromosomal locations and related gene sequences were also obtained from this website. Conserved domains were identified using Pfam (http://pfam.xfam.org/) [35, 36]. TMHMM3.0 was used for transmembrane domain prediction [37]. Multiple sequence alignment was performed, and a neighbor joining tree was created using ClustalW and MEGA 7, respectively.

\section{RNA extraction, CDNA synthesis, and qRT-PCR}

Total RNA was extracted with the BiozolTM Reagent (BioFlux, Tokyo, Japan) and treated with DNase I according to the manufacturer's instructions. Three $\mu \mathrm{g}$ of RNA were subjected to first strand cDNA synthesis with an oligo $(\mathrm{dT})_{18}$ primer using an RT-PCR system (Promega, Madison, WI, USA). Relative quantification of 
TaAMT2;3a expression was performed with a SYBR Green qRT-PCR mixture on an Biorad CFX Connect Real-Time PCR Detection System (Biorad, USA). Specific primers (Additional file 4: Table S2) were designed and qRT-PCR was conducted according to previously described procedures [38]. The wheat translation elongation factor TaEF-1a (GenBank Accession number Q03033) was used as an internal reference for all qRTPCR assays. All of the reactions were performed in triplicate using independent samples. The comparative $2^{-\Delta \Delta C T}$ method was used to quantify relative gene expression according to the Threshold values (Ct) [39].

\section{Subcellular location of TaAMT2:3a-GFP fusion protein}

To analyze the subcellular localization of TaAMT2;3a protein, the full length CDS of TaAMT2;3a was amplified by primers TaAMT2;3a(163)-F and TaAMT2; 3a(163)-R (Additional file 4: Table S2) and inserted into the PstI and BamHI sites of the pCAMV35S::GFP vector to generate the TaAMT2;3a-GFP fusion vector. The protoplasts were isolated from leaf tissue of one-week-old wheat seedlings as previously described [40]. The recombinant vector and the control plasmid pCAMV35S::GFP were transformed into wheat protoplasts by the PEGmediated transformation. The transfected mesophyll protoplasts were incubated in W5 solution in a dark chamber at $23^{\circ} \mathrm{C}$ for $18 \mathrm{~h}$. GFP fluorescence were observed with a Zeiss LSM700 confocal laser microscope (Zeiss, Germany) with a $480-\mathrm{nm}$ filter as previously described [41].

\section{Virus-induced gene silencing (VIGS) of TaAMT2;3a}

To analysis the function of TaAMT2;3a during wheatPst interaction, virus-induced gene silencing (VIGS) technology was used [42]. Two specific fragments (TaAMT2;3a-1as and TaAMT2;3a-2as) with no similarities to other wheat genes were selected to silence TaAMT2;3a (Additional file 4: Table S2). In vitro transcripts were prepared from the linearized plasmids containing the tripartite BSMV genome using the RiboMAX TM Large-Scale RNA Production System-T7 (Promega, Madison, WI, USA) and the Ribom7G Cap Analog (Promega), according to the manufacturer's instructions. The second leaves from wheat plants in two-leaf stage were infected with BSMV transcripts by rubbing inoculation and then incubated at $25^{\circ} \mathrm{C}$. BSMV: TaPDS was used as a positive control to confirm the successful genesilencing [42]. Control plants were treated with $1 \times$ FES buffer lacking of BSMV transcripts. Virus symptoms of the third leaves were observed and photographed at 10 days post-viral inoculation. The fourth leaves were then inoculated with CYR31 and sampled at 0, 24, 48, and 120 hpi for RNA isolation and histological observation. Wheat phenotypes after Pst inoculation were recorded and photographed at $14 \mathrm{dpi}$. The experiment was repeated three times and 20 plants were used for each fragment each time.

\section{Histological observations of fungal infection}

Wheat leaves infected with BSMV were sampled at 0 , 24, 48, and 120 hpi and stained by wheat germ agglutinin (WGA) conjugated to Alexa 488 (Invitrogen, Carlsbad, CA, USA) as previously described [43]. The fungal development in TaAMT2;3a-knockdown plants and control plants infected with Pst was observed by microscope. For each sample, at least 30 infection sites from three leaves were examined to assess the number of haustoria and hyphal branches, hyphal length and infection area using Olympus BX53 Digital Fluorescence Microscope and DP-BSW software (Olympus, Tokyo, Japan). Standard deviations and Student's t-test were applied for statistical analysis.

\section{Determination of ammonium contents in different tissues of wheat}

In order to analyze the levels and change of ammonium nitrogen contents in different tissues from wheat plants infected by Pst, wheat seedlings were cultivated by hydroponics method (Hoagland culture medium). Two weeks old wheat seedlings were inoculated with Pst race CYR31. Then the leaf, stem and root samples were collected at 7 time points: $0,12,24,36,48,72$ and $120 \mathrm{~h}$ post inoculation (hpi) as well as the non-inoculated control. Leaf samples from BSMV induced gene silencing plants and the control plants were collected at 7 time points the same as above samples. Ammonium was extracted by grinding frozen plant tissue samples into fine powder with a mortar under liquid N2 conditions. The $100 \mathrm{mg}$ tissue powder were then homogenized by $200 \mu \mathrm{l}$ extraction buffer $(50 \mathrm{~mm}$ Tris- $\mathrm{HCl}, \mathrm{pH} 8.0,10 \mathrm{~mm}$ imidazole, and $0.5 \%$ $[\mathrm{w} / \mathrm{v}] \quad \beta$-mercaptoethanol) in a $1.5 \mathrm{ml}$ microtube. After that, centrifuge the microtube at $12,000 \mathrm{~g}$ for $1 \mathrm{~min}$ at $4{ }^{\circ} \mathrm{C}$. Transfer the supernatant into a new $1.5 \mathrm{ml}$ microtube and the ammonium concentrations were determined by enzymatic method using an Ammonia Assay Kit (Sigma, Catalog Number: AA0100) according to the manufacturer's instructions. The relative ammonium concentration was calculated by subtracting the ammonium concentration of the non-inoculated control plants from that of the inoculated plants.

\section{Yeast complementation assay}

The TaATM2;3a CDS amplified by primers pDR195TaAMT2;3a-F and pDR195-TaAMT2;3a-R (Additional file 4: Table S2) was cloned into the HindIII site of yeast expression vector pDR195. The ammonium uptake deficient yeast strain 31019b (mep1, mep2, mep3, ura3) was obtained from the Bruno Andre (Univ Libre Bruxelles, 
Belgium). This strain lacks the endogenous ammonium transporters Mep1-3 and therefore is unable to grow on medium containing $<5 \mathrm{mM} \mathrm{NH}_{4}{ }^{+}$as the sole nitrogen source [44]. pDR195-TaATM2;3a and empty pDR195 (contains a yeast Ura 3 gene and acts as a negative control) were transformed into 31019b cells. The successful transformants were screened by growing of yeast cells in the SD/ -ura solid medium. Positive clones were pre-cultured in liquid yeast in the $\mathrm{SD} /$-ura medium until the absorbance value at $600 \mathrm{~nm}\left(\mathrm{OD}_{600}\right)$ reached 0.5 . Collect deposits were diluted by 10 times to $10^{-1}, 10^{-2}, 10^{-3}$ and $10^{-4}$ in $1 \times$ Tris-EDTA buffer. Then $6 \mu \mathrm{L}$ of each transformant was plated on yeast $\mathrm{N}$ base media containing $1 \mathrm{mM}, 5 \mathrm{mM}$ $\mathrm{NH}_{4} \mathrm{Cl}$ and $1 \mathrm{mM}$ Arginine, respectively. Yeast growth was monitored at $30^{\circ} \mathrm{C}$ for 3 days.

\section{Additional files}

Additional file 1: Figure S1 Sequence alignment of three homoeologs of TaAMT2;3a in wheat A, B and D genome. (TIF $12494 \mathrm{~kb}$ )

Additional file 2: Figure S2 The prediction of transmembrane region of wheat TaAMT2;3a by TMHMM3.0. (TIF 159 kb)

Additional file 3: Table S1. List of names, accession ID and protein sequences of ammonium transporters genes analyzed in this study. (XLSX $15 \mathrm{~kb}$ )

Additional file 4: Table S2 Information of primers used in this study. (XLSX $10 \mathrm{~kb})$

\section{Abbreviations}

AM: Arbuscular mycorrhizal; AMT: Ammonium transporter; Arg: Arginine; At: Arabidopsis thaliana; bp: Base-pairs; BSMV: Barley stripe mosaic virus; EHM: Extrahaustoria matrix; H: Haustorium; HMC: Haustorial mother cell; hpi: Hours post-inoculation; IH: Infection hyphae; Mp: Marchantid polymorpha; NIS: Nitrogen-induced susceptibility.; NRT: Nitrate transporter; ORF: Open reading frame; Os: Oryza sativa; PDS: Phytoene desaturase gene; Pgt: Puccinia graminis f. sp. tritici; Pst: Puccinia striiformis f. sp. tritici; qRTPCR: Quantitative realtime-PCR; SV: Substomatal vesicle; Ta: Triticum aestivum; VIGS: Virus-induced gene silencing; WGA: Wheat germ agglutinin
\end{abstract}

\section{Acknowledgements}

We thank Prof. Bruno Andre (Univ Libre Bruxelles, Belgium) for providing the yeast strain 31019b (mep1, mep2, mep3, ura3).

\section{Authors' contributions}

$J Z$, JF, and ZSK conceived and designed the experiment. JPJ, JZ, WLD, ST, XDW and $\mathrm{HZ}$ performed experiments. JPJ and JZ cloned the gene and performed the gene functional analysis. JPJ and $\mathrm{HZ}$ performed the expression pattern analysis. JPJ and WLD performed the experiment to assess the biochemical activity of protein. ST and XDW performed the protein subcellular localization analysis and histological observations. JZ, JF and ZSK analyzed the data and provided critical comments and suggestions on experiments. JPJ, JZ and JF wrote the paper. All the authors have seen and approved the manuscript in final version prior to submission.

\section{Funding}

This study was supported by the National Key Research and Development Program of China (2018YFD0200402) for writing and revising the manuscript, the National Transgenic Key Project of the Ministry of Agriculture of China (2016ZX08002-01) and the Research Funds for the Central Universities (2452017405) for designing and performing experiments.

\section{Availability of data and materials}

Data generated or analyzed in this study are included in this article and its supplementary information files.
Ethics approval and consent to participate

Not applicable.

\section{Consent for publication}

Not applicable.

\section{Competing interests}

The authors declare that they have no competing interests.

Received: 7 November 2018 Accepted: 21 May 2019

Published online: 06 June 2019

\section{References}

1. Tegeder M, Masclaux-Daubresse C. Source and sink mechanisms of nitrogen transport and use. New Phytol. 2018;217(1):35-53.

2. Tabuchi M, Abiko T, Yamaya T. Assimilation of ammonium ions and reutilization of nitrogen in rice (Oryza sativa L.). J Exp Bot. 2007:58(9): 2319-27.

3. Crawford NM, Forde BG. Molecular and developmental biology of inorganic nitrogen nutrition. Arabidopsis Book. 2002;1:e0011

4. Frink CR, Waggoner PE, Ausubel JH. Nitrogen fertilizer: retrospect and prospect. Proc Natl Acad Sci U S A. 1999;96(4):1175-80.

5. Fagard M, Launay A, Clement G, Courtial J, Dellagi A, Farjad M, Krapp A, Soulie MC, Masclaux-Daubresse C. Nitrogen metabolism meets phytopathology. J Exp Bot. 2014:65(19):5643-56.

6. Bajgain P, Russell B, Mohammadi M. Phylogenetic analyses and in-seedling expression of ammonium and nitrate transporters in wheat. Sci Rep. 2018;8: 7082

7. Xuan W, Beeckman T, Xu GH. Plant nitrogen nutrition: sensing and signaling. Curr Opin Plant Biol. 2017;39:57-65.

8. Mayer M, Dynowski M, Ludewig U. Ammonium ion transport by the AMT/ Rh homologue LeAMT1;1. Biochem J. 2006:396:431-7.

9. Yuan L, Loqué D, Kojima S, Rauch S, Ishiyama K, Inoue E, Takahashi H, Wirén NV. The Organization of High-Affinity Ammonium Uptake in Arabidopsis roots depends on the spatial arrangement and biochemical properties of AMT1-type transporters. Plant Cell. 2007:19(8):2636-52.

10. Sohlenkamp C, Wood CC, Roeb GW, Udvardi MK. Characterization of Arabidopsis AtAMT2, a high-affinity ammonium transporter of the plasma membrane. Plant Physiol. 2002;130(4):1788-96.

11. Huang H, Nguyen Thi Thu T, He X, Gravot A, Bernillon S, Ballini E, Morel JB. Increase of fungal pathogenicity and role of plant glutamine in nitrogeninduced susceptibility (NIS) to Rice blast. Front Plant Sci. 2017;8:265.

12. Li T, Liao K, Xu X, Gao Y, Wang Z, Zhu X, Jia B, Xuan Y. Wheat ammonium transporter (AMT) gene family: diversity and possible role in host-pathogen interaction with stem rust. Front Plant Sci. 2017:8:1637.

13. Dechorgnat J, Patrit O, Krapp A, Fagard M, Daniel-Vedele F. Characterization of the Nrt2.6 gene in Arabidopsis thaliana: a link with plant response to biotic and abiotic stress. PLoS One. 2012;7(8).

14. Voegele RT, Mendgen KW. Nutrient uptake in rust fungi: how sweet is parasitic life? Euphytica. 2011;179(1):41-55

15. Chang Q, Liu J, Lin XH, Hu SJ, Yang Y, Li D, Chen LY, Huai BY, Huang LL, Voegele RT, et al. A unique invertase is important for sugar absorption of an obligate biotrophic pathogen during infection. New Phytol. 2017;215(4): 1548-61.

16. Liu J, Han LN, Huai BY, Zheng PJ, Chang Q, Guan T, Li D, Huang LL, Kang ZS. Down-regulation of a wheat alkaline/neutral invertase correlates with reduced host susceptibility to wheat stripe rust caused by Puccinia striiformis. J Exp Bot. 2015:66(22):7325-38.

17. Voegele RT, Struck C, Hahn M, Mendgen K. The role of haustoria in sugar supply during infection of broad bean by the rust fungus Uromyces fabae. Proc Natl Acad Sci U S A. 2001;98(14):8133-8.

18. Neumann S, Paveley ND, Beed FD, Sylvester-Bradley R. Nitrogen per unit leaf area affects the upper asymptote of Puccinia striiformis f.sp tritici epidemics in winter wheat. Plant Pathol. 2004;53(6):725-32.

19. Robert C, Bancal MO, Lannou C. Wheat leaf rust uredospore production and carbon and nitrogen export in relation to lesion size and density. Phytopathology. 2002:92(7):762-8.

20. Dobon A, Bunting DC, Cabrera-Quio LE, Uauy C, Saunders DG. The hostpathogen interaction between wheat and yellow rust induces temporally coordinated waves of gene expression. BMC Genomics. 2016;17:380. 
21. Loque $D$, von Wiren $N$. Regulatory levels for the transport of ammonium in plant roots. J Exp Bot. 2004;55(401):1293-305.

22. Loque D, Lalonde S, Looger LL, von Wiren N, Frommer WB: A cytosolic trans-activation domain essential for ammonium uptake. Nature 2007: 446(7132):195-8.

23. Straub T, Ludewig U, Neuhaeuser B. The kinase CIPK23 inhibits ammonium transport in Arabidopsis thaliana. Plant Cell. 2017;29(2):409-22.

24. Lanquar V, Loque D, Hormann F, Yuan LX, Bohner A, Engelsberger WR, Lalonde S, Schulze WX, von Wiren N, Frommer WB. Feedback inhibition of ammonium uptake by a Phospho-dependent allosteric mechanism in Arabidopsis. Plant Cell. 2009;21(11):3610-22.

25. Guo H, Wang N, McDonald TR, Reinders A, Ward JM. MpAMT1;2 from Marchantia polymorpha is a high-affinity, plasma membrane ammonium transporter. Plant Cell Physiol. 2018;59(5):997-1005.

26. Giehl RFH, Laginha AM, Duan F, Rentsch D, Yuan L, von Wirén N. A critical role of AMT2;1 in root-to-shoot translocation of ammonium in Arabidopsis. Mol Plant. 2017:10(11):1449-60.

27. Chen AQ, Gu M, Wang SS, Chen JD, Xu GH. Transport properties and regulatory roles of nitrogen in arbuscular mycorrhizal symbiosis. Semin Cell Dev Biol. 2018;74:80-8

28. Koegel S, Lahmidi NA, Arnould C, Chatagnier O, Walder F, Ineichen K, Boller T, Wipf D, Wiemken A, Courty P-E. The family of ammonium transporters (AMT) in Sorghum bicolor: two AMT members are induced locally, but not systemically in roots colonized by arbuscular mycorrhizal fungi. New Phytol. 2013;198(3):853-65.

29. Koegel S, Mieulet D, Baday S, Chatagnier O, Lehmann MF, Wiemken A, Boller T, Wipf D, Berneche S, Guiderdoni E, et al. Phylogenetic, structural, and functional characterization of AMT3;1, an ammonium transporter induced by mycorrhization among model grasses. Mycorrhiza. 2017;27(7): 695-708.

30. Breuillin-Sessoms F, Floss DS, Gomez SK, Pumplin N, Ding Y, LevesqueTremblay V, Noar RD, Daniels DA, Bravo A, Eaglesham JB, et al. Suppression of Arbuscule degeneration in Medicago truncatula phosphate transporter4 mutants is dependent on the ammonium transporter 2 family protein AMT2;3. Plant Cell. 2015;27(4):1352-66.

31. Pastor V, Gamir J, Camañes G, Cerezo M, Sánchez-Bel P, Flors V. Disruption of the ammonium transporter AMT1.1 alters basal defenses generating resistance against Pseudomonas syringae and Plectosphaerella cucumerina. Front Plant Sci. 2014;5:231

32. Schwessinger B, Sperschneider J, Cuddy WS, Garnica DP, Miller ME, Taylor JM, Dodds PN, Figueroa M, Park RF, Rathjen JP. A near-complete haplotypephased genome of the Dikaryotic wheat stripe rust fungus Puccinia striiformis f. sp tritici reveals high Interhaplotype diversity. mBio. 2018;9(1): e02275-17. https://doi.org/10.1128/mBio.02275-17.

33. Kang Z, Pirskanen A, Janne OA, Palvimo JJ. Involvement of proteasome in the dynamic assembly of the androgen receptor transcription complex. J Biol Chem. 2002;277(50):48366-71.

34. Tang C, Deng L, Chang D, Chen S, Wang X, Kang Z. TaADF3, an actindepolymerizing factor, negatively modulates wheat resistance against Puccinia striiformis. Front Plant Sci. 2015;6:1214.

35. Ahmed SM, Liu P, Xue Q, Ji C, Qi T, Guo J, Guo J, Kang Z. TaDIR1-2, a wheat Ortholog of lipid transfer protein AtDIR1 contributes to negative regulation of wheat resistance against Puccinia striiformis f. sp. tritici. Front Plant Sci. 2017;8:521.

36. Jones $P$, Binns D, Chang H-Y, Fraser M, Li W, McAnulla C, McWilliam H, Maslen J, Mitchell A, Nuka G, et al. InterProScan 5: genome-scale protein function classification. Bioinformatics. 2014;30(9):1236-40

37. Wang X, Wang X, Deng L, Chang H, Dubcovsky J, Feng H, Han Q, Huang L, Kang Z. Wheat TaNPSN SNARE homologues are involved in vesiclemediated resistance to stripe rust (Puccinia striiformis f. sp. tritici). J Exp Bot. 2014;65(17):4807-20

38. Wang X, Tang C, Zhang G, Li Y, Wang C, Liu B, Qu Z, Zhao J, Han Q, Huang $L$, et al. CDNA-AFLP analysis reveals differential gene expression in compatible interaction of wheat challenged with Puccinia striiformis f. sp. tritici. BMC Genomics. 2009;10:289.

39. Livak KJ, Schmittgen TD. Analysis of relative gene expression data using real-time quantitative PCR and the $2-\Delta \Delta C T$ method. Methods. 2001;25(4): 402-8.

40. Liu P, Myo T, Ma W, Lan D, Qi T, Guo J, Song P, Guo J, Kang Z. TaTypA, a ribosome-binding GTPase protein, positively regulates wheat resistance to the stripe rust fungus. Front Plant Sci. 2016;7:873.
41. Ito T, Shinozaki K. The MALE STERILITY1 gene of Arabidopsis, encoding a nuclear protein with a PHD-finger motif, is expressed in tapetal cells and is required for pollen maturation. Plant Cell Physiol. 2002;43(11):1285-92.

42. Holzberg S, Brosio P, Gross C, Pogue GP. Barley stripe mosaic virus-induced gene silencing in a monocot plant. Plant J. 2002;30(3):315-27.

43. Ayliffe M, Devilla R, Mago R, White R, Talbot M, Pryor A, Leung H. Nonhost resistance of rice to rust pathogens. Mol Plant-Microbe Interact. 2011;24(10): 1143-55.

44. Marini AM, Soussi-Boudekou S, Vissers S, Andre B. A family of ammonium transporters in Saccharomyces cerevisiae. Mol Cell Biol. 1997;17(8):4282-93.

\section{Publisher's Note}

Springer Nature remains neutral with regard to jurisdictional claims in published maps and institutional affiliations.
Ready to submit your research? Choose BMC and benefit from:

- fast, convenient online submission

- thorough peer review by experienced researchers in your field

- rapid publication on acceptance

- support for research data, including large and complex data types

- gold Open Access which fosters wider collaboration and increased citations

- maximum visibility for your research: over $100 \mathrm{M}$ website views per year

At $\mathrm{BMC}$, research is always in progress.

Learn more biomedcentral.com/submissions 